\title{
In which patients is transcatheter aortic valve replacement potentially better indicated than surgery for redo aortic valve disease? Long-term results of a 10-year surgical experience
}

\author{
Konstantinos Pechlivanidis, MD, Francesco Onorati, MD, PhD, Giuseppe Petrilli, MD, \\ Francesco Santini, MD, Aldo Milano, MD, Salvatore Torre, MD, Daniele Calzaferri, MD, \\ Alessandro Mazzucco, MD, and Giuseppe Faggian, MD
}

\begin{abstract}
Background: Redo aortic valve replacement procedures have been reduced by the growing practice of trans-catheter aortic valve-in-valve procedures. We analyzed our long-term results of redo aortic valve replacement procedures during a 10-year period in an effort to define subgroups in which trans-catheter aortic valve-in-valve procedures may be better than surgery.
\end{abstract}

\begin{abstract}
Methods: From 2002 to 2010, 131 redo aortic valve replacement procedures with at least 18 months of follow-up were prospectively enrolled. Hospital and follow-up outcome of the entire population and of high-risk subgroups were evaluated.
\end{abstract}

\begin{abstract}
Results: Hospital mortality was $2.3 \%$, major re-entry complications were seen in $1.5 \%$, re-exploration for bleeding was seen in $9.2 \%$, perioperative low cardiac output state (ie, low cardiac output syndrome) was seen in $9.9 \%$, stroke was seen in $3.1 \%$, prolonged ventilation was seen in $18.3 \%$, pneumonia was seen in $4.6 \%$, acute renal insufficiency was seen in $11.5 \%$, intra-aortic counterpulsation (intra-aortic balloon pump) was seen in $9.2 \%$, renal replacement therapy was seen in $4.6 \%$, need for transfusions was seen in $60.3 \%$, and permanent pacemaker implantation was seen in $2.3 \%$. One hundred twenty-month actuarial survival, freedom from acute heart failure, reinterventions, stroke, and thromboembolisms were $61.5 \% \pm 8.6 \%$, $62.9 \% \pm 6.9 \%, 97.8 \% \pm 1.5 \%, 93.2 \% \pm 3.0 \%$, and $91.2 \% \pm 3.2 \%$, respectively. Patients aged $>75$ years had similar outcome to younger patients (nonsignificant $P$ for all). Endocarditis resulted in higher hospital mortality $(P=.034)$, low cardiac output state $(P<.0001)$, intra-aortic balloon pump $(P<.0001)$, prolonged ventilation $(P=.011)$, pneumonia $(P=.049)$, acute renal insufficiency $(P=.004)$, lower actuarial survival (log-rank $P=.0001)$, freedom from acute heart failure $(P=.002)$, and re-intervention $(P=.003)$. New York Heart Association functional class IV at admission resulted in a higher incidence of low cardiac output state $(P<.0001)$, intra-aortic balloon pump $(P=.0001)$, prolonged ventilation $(P<.0001)$, pneumonia $(P=.015)$, and a lower actuarial freedom from re-intervention $(P=.0001)$. Higher need for permanent pacemaker implantation $(P=.015)$ and lower freedom from acute heart failure $(P=.019)$ emerged after urgencies/emergencies.

Conclusions: Redo aortic valve replacement procedures achieves good results, especially in nonendocarditic or elective cases, and young or New York Heart Association functional class I/II patients. Indeed, endocarditis significantly affects outcome. New York Heart Association functional class IV and nonelective procedures might benefit from trans-catheter aortic valve-in-valve procedures. (J Thorac Cardiovasc Surg 2014;148:500-8)
\end{abstract}

Supplemental material is available online.

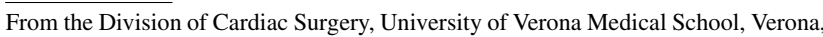
Italy.

Disclosures: Authors have nothing to disclose with regard to commercial support. Received for publication Feb 26, 2013; revisions received Aug 1, 2013; accepted for publication Sept 10, 2013; available ahead of print Nov 1, 2013.

Address for reprints: Francesco Onorati, MD, PhD, Division of Cardiac Surgery, University of Verona Medical School, Piazzale Stefani, 1, 37126, Verona, Italy (E-mail: frankono@libero.it).

0022-5223/\$36.00

Copyright (c) 2014 by The American Association for Thoracic Surgery

http://dx.doi.org/10.1016/j.jtcvs.2013.09.031
}

Aortic valve replacement (AVR) is the most frequently employed cardiac valve operation, with more than 85,000 procedures performed yearly in the United States. ${ }^{1}$ With the exponential growth of the geriatric population, the need for redo AVR (RAVR) is similarly growing. ${ }^{2-4}$ Indeed, any redo cardiac surgery increases the risk of mortality and morbidity when compared with the corresponding first-time operation. ${ }^{2-7}$ However, the extremely variable risk of mortality reported after RAVR has been attributed to different factors related to the risk profile of the enrolled population, the operator's skill, and the surgical volume of individual hospitals. ${ }^{2-7}$ Furthermore, the very high mortality rate reported in some surgical experiences mandates identification of 


\section{Abbreviations and Acronymns \\ AVR = aortic valve replacement \\ EuroSCORE $=$ European System for Cardiac \\ Operative Risk Evaluation \\ NYHA = New York Heart Association \\ RAVR $\quad$ redo aortic valve repair \\ TAVIV $=$ transcatheter aortic valve-in-valve}

peculiar very-high-risk subgroups. ${ }^{2-7}$ On the other hand, the fragile profile of the geriatric population has led in past years to the widespread diffusion of transcatheter aortic valve-in-valve (TAVIV) procedures, given a reported relatively low risk of hospital mortality and morbidity. ${ }^{8,9}$ However, midterm and late outcome of TAVIV are still unknown. ${ }^{8,9}$

Therefore, it was the aim of this study to analyze in-hospital and long-term results of a consecutive series of patients, and of specific high-risk subgroups, with at least 18 months of follow-up, undergoing RAVR during the past 10 years at our large-volume institution, to serve as benchmark for RAVR and to define subgroups in which TAVIV may be better than surgery or contraindicated.

\section{METHODS}

\section{Scope and Data Collection}

It was the aim of our study to evaluate the outcome of isolated RAVR or RAVR plus cardiopulmonary artery bypass graft in patients considered potentially eligible - in the current era-for TAVIV. Therefore, patients with concomitant root or mitral valve disease were not enrolled in the study. Accordingly, given the possibility for concomitant percutaneous coronary intervention during TAVIV, patients undergoing RAVR with concomitant coronary artery bypass graft were considered eligible for enrollment. Accordingly, 131 consecutive RAVR (with or without concomitant coronary artery bypass graft) patients admitted to our institution from January 2002 to June 2011, with at least 18 months of follow-up, were enrolled.

The choice to start enrollment from 2002 was done to avoid potential biases related to differences in perioperative management and care, as well as to have a picture of current RAVR practice at our institution. Follow-up was closed on December 30, 2012; therefore, the choice to truncate the enrollment at June 2011 was dictated by the intention to evaluate at least midterm outcome at 18 months. No patient was lost during follow-up, which was therefore $100 \%$ complete.

High-risk subgroups were chosen based on traditional risk factors for hospital mortality according to the European System for Cardiac Operative Risk Evaluation (EuroSCORE) score, as well as to worldwide accepted literature data, ${ }^{1-4,10}$ provided that an acceptable sample size was at least detected for each risk group in our population. Therefore, elderly patients (aged $>75$ years), patients admitted in New York Heart Association (NYHA) functional class IV, urgent/emergent procedures, and endocarditic etiology were specifically analyzed. On the other hand, to define patients at potential very-low-risk after RAVR, subgroups of young patients (aged $<65$ years) and those admitted in NYHA functional class I or II were specifically investigated in terms of long-term outcome.

All data were collected prospectively in the institutional database and hospital charts but retrospectively analyzed. It was our institution's policy to discharge all patients to rehabilitation clinics; thus, follow-up started at our outpatient clinic at the end of the rehabilitation program, where patients were followed-up with at least once. Events that occurred during rehabilitation were collected from rehabilitation hospital charts and follow-up was then continued by cardiac surgeons at the outpatient clinic by the referral cardiologist or by the patient's general practitioner after the first surgical control. Follow-up data collection was based on patient charts of our outpatient clinic, on telephone contacts with cardiologists or general practitioners, and finally - in the absence of recent data- by direct telephone contact with the patient. Institutional review board approval and individual patient consent were waived due to the observational nature of the study.

\section{Surgery}

The choice for mechanical or biological prosthesis was left to patient preference after discussion and evaluation with a surgeon regarding the risks and benefits of each choice. Anesthesia, surgery, and cardiopulmonary bypass were standardized and reported elsewhere. ${ }^{11}$ Preoperative chest computed tomography was performed in all patients per institutional policy, to correctly plan surgical re-entry. Surgical access consisted in a median full re-sternotomy in all patients, and no ministernotomy or alternative accesses were ever used during the study period. Peripheral cannulation was chosen when surgical re-entry via median re-sternotomy was considered high risk, but no percutaneous peripheral cannulation was employed during the study period. Postoperative care was similarly standardized and already reported. ${ }^{11}$

\section{Definitions and Endpoints}

Primary endpoints of the study were hospital mortality, which was defined as all-cause mortality during the index hospitalization, and follow-up mortality. The secondary endpoints were rate of hospital complications, and follow-up freedom from acute heart failure, reoperation, stroke, and thromboembolisms. ${ }^{11,12}$

The following hospital complications were collected: major cardiovascular re-entry complications, defined as any severe and/or life-threatening (ie, requiring reanimation and/or immediate changing of the surgical plan and/or massive transfusions $>4$ red packed cells) injury of major vessels or cardiac structures, occurred during surgical re-entry; revision for bleeding, defined as any reoperation during the index hospitalization, due to postoperative bleeding; need for permanent pacemaker implantation; low cardiac output syndrome, defined as hemodynamic instability for $>1$ hour during the intensive care unit stay, with peripheral signs of hypoperfusion despite inotropic support and adequate correction of preload, afterload, and all electrolyte and blood gas abnormalities ${ }^{11}$; need for intraoperative/postoperative intra-aortic balloon pump; prolonged intubation, defined as the need for prolonged ( $>48$ hours) mechanical ventilation or acute respiratory insufficiency after extubation with need for reintubation or need for noninvasive ventilation lasting $>48$ hours $^{11}$; pneumonia, defined as evidence of bacterial growth in the lung with at least 1 positive bronchoalveolar fluid lavage culture, together with new alveolar infiltrates at chest roentgenogram, irrespective of the presence of fever or leukocytosis, or as evidence of new alveolar infiltrates with leukocytosis and purulent sputum, confirmed by computed tomography scan and/or by consultation of an independent infectivologist or pneumologist ${ }^{11}$; stroke, defined per current guidelines ${ }^{12}$; and acute renal insufficiency, defined as a $>50 \%$ increase over the preoperative serum creatinine value. ${ }^{11}$ Other perioperative collected variables considered as surrogates of the quality of clinical outcome were length of intubation (expressed in hours), need for transfusions (regardless of red packed cells, fresh frozen plasma, or platelets), and length of hospitalization (expressed in days, starting from the day of surgery).

Apart from survival, other outcome variables collected during follow-up were acute heart failure, defined as any episode of acute congestive heart failure requiring hospitalization and/or optimization of medical therapy; reintervention, defined as any reoperation on the aortic valve prosthesis 
implanted at the time of the indexed RAVR; stroke, as defined above; and thromboembolisms, defined per current guidelines. ${ }^{12}$

Finally, when baseline characteristics were considered, urgent/emergent operations were defined as operative procedures for life-threatening conditions performed within 24 hours from hospital admission.

\section{Statistical Analysis}

Continuous variables are presented as mean \pm standard deviation, and categorical variables are presented as absolute numbers and percentages. Data were checked for normality before statistical analysis. Normally distributed continuous variables were compared using the unpaired $t$ test, whereas the Mann-Whitney $U$ test was used for non-normally distributed variables. Categorical variables were analyzed using either the $\chi^{2}$ test or Fischer exact test as appropriate.

Due to the limited number of events at follow-up, a composite endpoint of follow-up mortality and/or acute heart failure was considered. Because of the intention to identify baseline characteristics predicting a high risk of follow-up events after RAVR - to suggest alternative procedures to RAVR (eg, transcatheter aortic valve replacement)—only preoperative variables (including planned surgical intervention) were subjected to univariate analysis for the composite endpoint. Variables with a $P$ value of .10 or less in the univariate analysis were consecutively subjected to a multivariate logistic regression model to assess the independent influence of each risk factor on the selected outcome. In detail, 6 preoperative/intraoperative variables were selected to enter the multivariate model. A stepwise (backward Wald) multivariable binary logistic regression analysis was used. Both regression model discrimination and calibration were assessed by using the $C$ statistic for regression model discrimination and the Hosmer-Lemeshow test statistic for model calibration. The models were expressed in terms of adjusted odds ratio, $95 \%$ confidence interval, and $P$ value. Follow-up mortality and freedom from events were determined with the method of Kaplan-Meier life table analysis. Log-rank test was performed to ascertain between-groups differences. Statistical analysis was performed by the SPSS program for Windows, version 13.0 (IBM-SPSS Inc, Armonk, NY).

\section{RESULTS}

Preoperative and operative characteristics of 131 enrolled patients are reported in Table E1. The proportion of investigated high-risk subgroups were: $26.7 \%$ elderly patients aged $>75$ years (35 patients), $15.3 \%$ patients admitted in NYHA functional class IV (20 patients), $334.4 \%$ urgent/emergent priority admission patients (45 patients), and $19.1 \%$ endocarditic etiology patients (25 patients).

\section{Hospital and Follow-up Outcome in the Entire Population}

Hospital mortality was $2.3 \%$ ( 3 patients). The reported rates of hospital complications were: major re-entry complications $1.5 \%$, revision for bleeding $9.2 \%$, need for permanent pacemaker $2.3 \%$, low-output cardiac state $9.9 \%$, intra-aortic counterpulsation (intra-aortic balloon pump) $9.2 \%$, stroke $3.1 \%$, prolonged ventilation $18.3 \%$, pneumonia $4.6 \%$, acute renal insufficiency $11.5 \%$, renal replacement therapy $4.6 \%$, and need for transfusions $60.3 \%$. Mean follow-up was $55.1 \pm 32.4$ months. Ten-year survival was $61.5 \% \pm 8.6 \%$. Ten-year freedom from acute heart failure, reoperations, stroke, and thromboembolisms were $62.9 \% \pm 6.9 \%, 97.8 \% \pm 1.5 \%, 93.2 \% \pm 3.0 \%$, and $91.2 \% \pm 3.2 \%$, respectively (Figure 1).

\section{Hospital and Follow-up Outcome in Subgroups at Higher Risk}

When elderly patients were considered, hospital mortality and complications proved comparable to those of younger patients, although rough data indicate a slightly worse hospital outcome in the aged population (Table 1). An obvious lower (although not statistically significant) follow-up survival was detected (patients aged $>75$ years $52.5 \% \pm 11.3 \%$ vs patients aged $<75$ years $67.4 \% \pm$ $9.3 \% ; P=.168)$. Follow-up freedom from acute heart failure (patients aged $>75$ years $71.1 \% \pm 10.0 \%$ vs patients aged $<75$ years $61.7 \% \pm 7.9 \% ; P=.601$ ), reoperation (patients aged $>75$ years $100.0 \%$ vs patients aged $<75$ years $96.9 \% \pm 2.2 \% ; P=.367$ ), stroke (patients aged $>75$ years $90.9 \% \pm 8.7 \%$ vs patients aged $<75$ years $93.5 \% \pm 3.0 \% ; P=.581$ ) and thromboembolism (patients aged $>75$ years $88.3 \% \pm 8.8 \%$ vs patients aged $<75$ years $91.7 \% \pm 3.4 \% ; P=.898)$ proved similar, regardless of age. However, when young patients (aged $<65$ years) were analyzed, a particularly favorable long-term outcome was identified, with a $77.9 \% \pm 6.7 \% 10$-year survival, $70.1 \% \pm 5.8 \%$ freedom from acute heart failure, $96.7 \%$ $\pm 3.3 \%$ freedom from reoperation, $90.8 \% \pm 4.8 \%$ freedom from stroke, and $93.3 \% \pm 4.8 \%$ freedom from thromboembolisms.

When stratified by NYHA functional class, patients admitted in NYHA functional class IV showed a similar hospital mortality $(5.0 \%$ vs NYHA class $<\mathrm{IV}=1.8 \%)$, but also a significantly higher incidence of postoperative low cardiac output syndrome, need for intra-aortic balloon pump, prolonged intubation, and pneumonia (Table 1). When follow-up outcome was considered, survival proved comparable (NYHA class IV $=62.2 \% \pm 11.7 \%$ vs NYHA class $<$ IV $60.3 \% \pm 10.6 \% ; P=.159$ ), as well as stroke (Figure 2, C), and thromboembolism (Figure 2, D), whereas a lower but not significant freedom from acute heart failure was reported (Figure 2, A). Only freedom from reoperation was significantly lower in patients admitted with NYHA functional class IV $(P<.0001)$ (Figure 2, B). On the other hand, NYHA functional class I or II at admission identifies a subgroup of patients with an excellent follow-up outcome, given the reported $85.8 \% \pm 7.8 \%$ 10-year survival, $89.8 \% \pm 5.8 \%$ freedom from acute heart failure, $100 \%$ freedom from reintervention, $97.5 \% \pm 2.5 \%$ freedom from stroke, and $94.0 \% \pm 4.2 \%$ freedom from thromboembolisms.

When priority at admission was considered, those referred as emergent/urgent patients showed a comparable hospital outcome compared to elective cases, with the only exception being a higher need for permanent pacemaker implantation in the urgencies/emergencies 


\section{GLOBAL POPULATION}
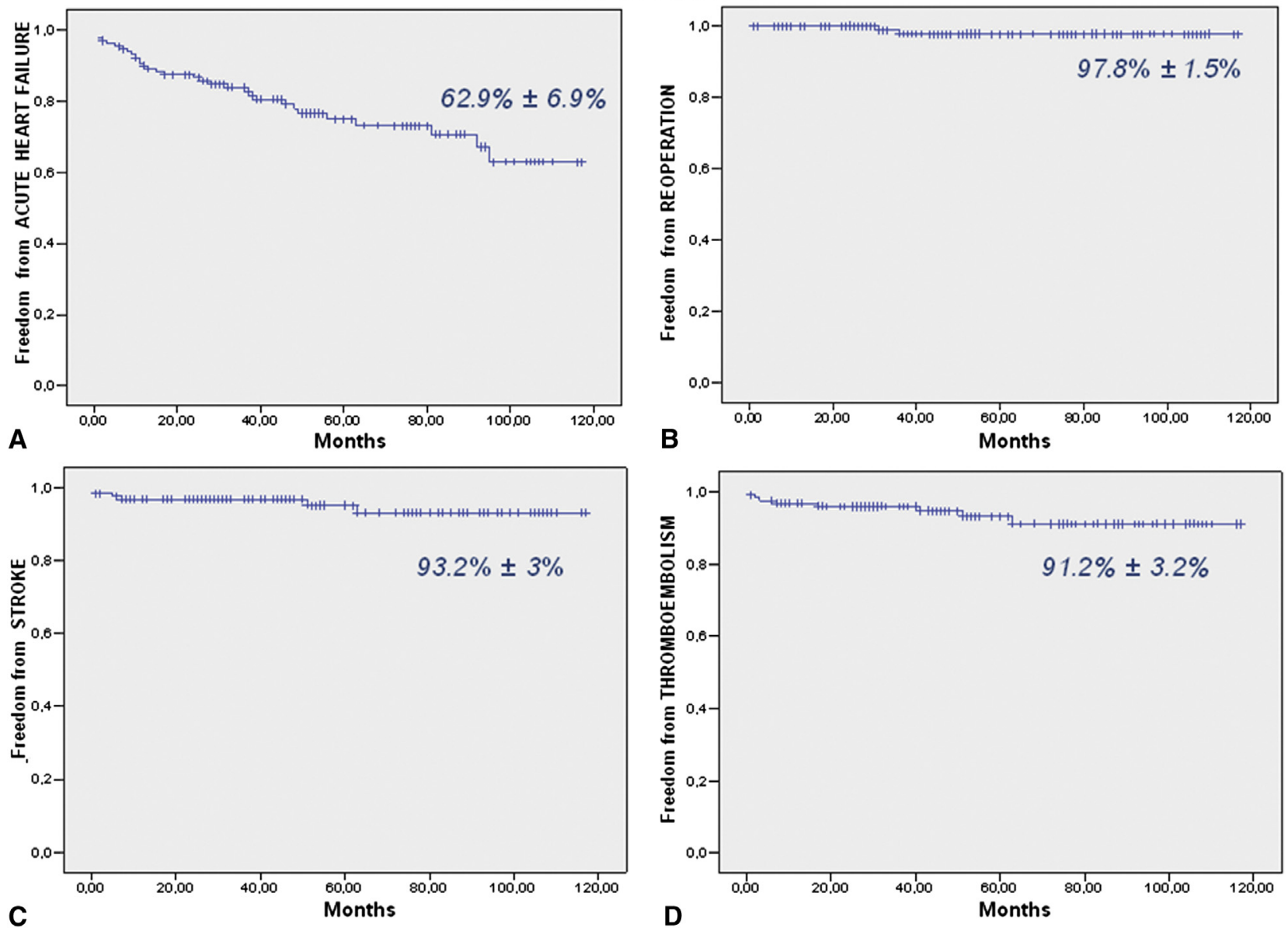

FIGURE 1. Ten-year actuarial freedom from (A) acute heart failure, (B) reoperations, (C) stroke, and (D) thromboembolisms.

(Table 1). Follow-up survival was lower, although not statistically significant in nonelective procedures (emergent/urgent $47.8 \% \pm 12.8 \%$ vs elective $66.8 \% \pm$ $10.0 \% ; P=.211)$. However, freedom from acute heart failure was significantly lower in urgent/emergent patients $(54.5 \% \pm 10.0 \%$ vs elective $68.8 \% \pm 8.8 \% ; P=.019)$ (Figure 3, A), whereas freedom from reoperation, stroke, and thromboembolism were similar between the 2 groups (Figure 3, B-D).

Finally, when the endocarditic etiology was considered, a significantly higher hospital mortality, prevalence of postoperative low cardiac output state and need for intraaortic balloon pump, presence of prolonged intubation and pneumonia, as well as of acute renal insufficiency were found in this cohort (Table 1). The other hospital outcome variables proved similar between the endocarditic and the nonendocarditic etiology (Table 1). Follow-up outcome was similarly worse for endocarditis, given the lower survival $(20.6 \% \pm 9.7 \%$ vs nonendocarditis $75.1 \%$ $\pm 12.0 \% ; P<.0001)$, freedom from acute heart failure $(P=.002)$ (Figure $4, A)$, and freedom from reoperation $(P=.003)$ (Figure $4, B)$. However, freedom from stroke and thromboembolism were similar (Figure 4, $C$ and $D$, respectively). On the other hand, the absence of endocarditis at RAVR identifies a subset of patients with a particularly favorable follow-up outcome $(75.1 \% \pm 12.0 \%$ 10-year survival, $67.7 \% \pm 7.6 \%$ freedom from acute heart failure, $100 \%$ freedom from reintervention, $90.9 \% \pm 8.7 \%$ freedom from stroke, and $86.4 \% \pm 9.4 \%$ freedom from thromboembolisms).

\section{Predictors of Composite Endpoint of Follow-up Mortality/Acute Heart Failure}

The following preoperative and intraoperative variables were significantly associated with the composite endpoint of follow-up mortality/acute heart failure at univariate analysis: EuroSCORE $(P=.0001)$, age $(P=.04)$, NYHA functional class at admission $(P=.0001)$, endocarditis $(P<.0001)$, major arrhythmias at admission $(P=.02)$, and planned surgical intervention $(P=.02)$. Of these, independent predictors of the composite endpoint of follow-up mortality/acute heart failure were EuroSCORE (odds ratio $[\mathrm{OR}]=1.3 ; 95 \%$ confidence interval $[\mathrm{CI}]$, 1.0-1.7; $P=.04)$, NYHA functional class at admission 
TABLE 1. Hospital outcome in subgroups at risk

\begin{tabular}{|c|c|c|c|c|c|c|c|c|c|c|c|c|}
\hline Variable & $\begin{array}{c}\text { Age } \\
>75 y \\
(n=35) \\
\end{array}$ & $\begin{array}{c}\text { Age } \\
<75 \mathrm{y} \\
(\mathrm{n}=96) \\
\end{array}$ & $\boldsymbol{P}$ & $\begin{array}{l}\text { NYHA } \\
\text { class IV } \\
(n=20)\end{array}$ & $\begin{array}{c}\text { NYHA } \\
\text { class I-III } \\
(n=111) \\
\end{array}$ & $P$ & $\begin{array}{c}\text { Urgent/ } \\
\text { Emergent } \\
(n=45) \\
\end{array}$ & $\begin{array}{l}\text { Elective } \\
(\mathbf{n}=\mathbf{8 6})\end{array}$ & $\boldsymbol{P}$ & $\begin{array}{c}\text { Endocarditis } \\
\quad(\mathbf{n}=\mathbf{2 5})\end{array}$ & $\begin{array}{c}\text { No } \\
\text { Endocarditis } \\
(\mathbf{n}=106) \\
\end{array}$ & $\boldsymbol{P}$ \\
\hline Mortality & $2.9(1)$ & $2.1(2)$ & .793 & $5.0(1)$ & $1.8(2)$ & .379 & $2.2(1)$ & $2.3(2)$ & .970 & $8.0(2)$ & $0.9(1)$ & .034 \\
\hline $\begin{array}{l}\text { Major cardiovascular } \\
\text { re-entry complications }\end{array}$ & $2.9(1)$ & $1.0(1)$ & .453 & $0(-)$ & $1.8(2)$ & .545 & $2.2(1)$ & $1.2(1)$ & .639 & $4.0(1)$ & $0.9(1)$ & .262 \\
\hline Revision for bleeding & $11.4(4)$ & $8.3(8)$ & .587 & $15.0(3)$ & $8.1(9)$ & .325 & $11.1(5)$ & $8.1(7)$ & .576 & $16.0(4)$ & $7.5(8)$ & .188 \\
\hline $\begin{array}{l}\text { Need for permanent } \\
\text { pacemaker }\end{array}$ & $5.7(2)$ & $1.0(1)$ & .114 & $0(-)$ & $2.7(3)$ & .457 & $6.7(3)$ & $0(-)$ & .015 & $4.0(1)$ & $1.9(2)$ & .525 \\
\hline Low cardiac output state & $11.4(4)$ & $9.4(9)$ & .728 & $40.0(8)$ & $4.5(5)$ & $<.001$ & $11.1(5)$ & $9.3(8)$ & .742 & $32.0(8)$ & $4.7(5)$ & $<.001$ \\
\hline $\begin{array}{l}\text { Intraoperative/postoperative } \\
\text { intra-aortic balloon pump }\end{array}$ & $8.6(3)$ & $9.4(9)$ & .888 & $35.0(7)$ & $4.5(5)$ & $<.001$ & $11.1(5)$ & $8.1(7)$ & .576 & $32.0(8)$ & $3.8(4)$ & $<.001$ \\
\hline Prolonged intubation ( $>48 \mathrm{~h}$ ) & $14.3(5)$ & $19.8(19)$ & .471 & $60.0(12)$ & $10.8(12)$ & $<.001$ & $20.0(9)$ & $17.4(15)$ & .719 & $36.0(9)$ & $14.2(15)$ & 011 \\
\hline Pneumonia & $8.6(3)$ & $3.1(3)$ & .187 & $15.0(3)$ & $2.7(3)$ & .015 & $6.7(3)$ & $3.5(3)$ & .409 & $12.0(3)$ & $2.8(3)$ & .049 \\
\hline Stroke & $2.9(1)$ & $3.1(3)$ & .937 & $5.0(1)$ & $2.7(3)$ & .583 & $0(0)$ & $4.7(4)$ & .142 & $4.0(1)$ & $2.8(3)$ & .760 \\
\hline Acute renal insufficiency & $11.4(4)$ & $11.5(11)$ & .996 & $20.0(4)$ & $9.9(11)$ & .192 & $13.3(6)$ & $10.5(9)$ & .624 & $28.0(7)$ & $7.5(8)$ & .004 \\
\hline Length of intubation, $h$ & $40.8 \pm 89.9$ & $23.3 \pm 43.5$ & .227 & $42.8 \pm 107.8$ & $25.6 \pm 47.7$ & .344 & $28.1 \pm 51.6$ & $28.1 \pm 64.2$ & .998 & $10.4 \pm 8.1$ & $29.3 \pm 61.7$ & .499 \\
\hline Transfused patients & $65.7(23)$ & $58.3(56)$ & .445 & $70.0(14)$ & $58.6(65)$ & .336 & $68.9(31)$ & $55.8(48)$ & .146 & $68.0(17)$ & $58.5(62)$ & .382 \\
\hline Length of hospitalization, $\mathrm{d}$ & $12.1 \pm 5.9$ & $12.5 \pm 7.1$ & .770 & $12.3 \pm 4.8$ & $12.4 \pm 7.0$ & .947 & $12.6 \pm 5.5$ & $12.3 \pm 7.4$ & .797 & $10.5 \pm 2.4$ & $12.5 \pm 7.0$ & .414 \\
\hline
\end{tabular}

Values are presented as mean \pm standard deviation or \% (n). NYHA, New York Heart Association.

$(\mathrm{OR}=2.1 ; 95 \%$ CI, 1.1-3.9; $P=.02)$, and endocarditic etiology $(\mathrm{OR}=18.0 ; 95 \% \mathrm{CI}, 4.6-61.2 ; P=.0001)$. The discrimination $C$ statistic for the final logistic regression analysis was .79 . The $\chi^{2}$ test result for the Hosmer-Lemeshow goodness of fit test was 6.7 for an overall $\chi^{2}$ significance of .680.

\section{DISCUSSION}

The current widespread use of transthoracic AVR, together with a reported high risk of perioperative mortality and major complications in redo aortic valve surgery, has led to an exponential use of transthoracic AVR technology for patients with aortic prosthetic dysfunction. ${ }^{9,13}$ In particular, some comorbid conditions and advanced age have been emphasized as preferential indications for TAVIV. ${ }^{9,13}$ On the other hand, current RAVR outcomes are a poorly addressed issue in the literature due to the fact that current reports of RAVR involve a case-mixed population of patients. ${ }^{3,4,6,7}$ Other studies focusing on the detrimental prognostic role of endocarditis on long-term outcome after RAVR have similarly reported very poor surgical results, ${ }^{10}$ although endocarditis is still a well-defined contraindication to TAVIV. Nevertheless, application of transthoracic AVR has been recently reported even in patients with active endocarditis. ${ }^{14}$ Furthermore, some other literature studies on TAVIV reported only early or short-term outcome ${ }^{15,16}$ or a limited number of patients. ${ }^{13,15}$ Therefore, the literature lacks studies addressing the long-term outcome of RAVR in a significant number of patients potentially eligible today for TAVIV procedure, and the relative roles of TAVIV and RAVR in that scenario.

We were able to show acceptable results, provided that endocarditic etiology is not reported. In particular, we found hospital mortality as low as $2.3 \%$, which was also significantly lower than what was predicted by our patientspecific preoperative EuroSCORE. Despite the fact that no direct comparison with TAVIV was considered in this study due to the lack of concomitant TAVIV experience at our institution, TAVIV has been reported to achieve a $0 \%$ hospital mortality in some centers, ${ }^{13}$ but also a $14 \%$ to $25 \%$ postoperative mortality in other experiences. ${ }^{17,18}$ Overall, a volume-related effect on outcome after TAVIV has been emphasized by recent studies, with results ranging from $100 \%$ to $66 \%{ }^{13}$ For example, Piazza and colleagues $^{19}$ reported an $88.0 \%$ procedural success with Sapien valves (Edwards Lifesciences, Irvine, Calif) and a $10 \% 30$-day mortality. Seiffert and colleagues ${ }^{18}$ reported a $25 \%$ 30-day mortality in 4 patients undergoing TAVIV. It should be emphasized that all these reports demonstrated excellent early results with TAVIV in patients unsuitable for traditional surgical RAVR. ${ }^{13,18,19}$

On the other hand, a recent Canadian experience on a very large number of case-mixed cardiac redo surgical procedures reported an overall hospital mortality $<6 \%$, further reduced to $4 \%$ in isolated RAVR, and concluded that more favorable results than what currently predicted by risk scores can be expected. ${ }^{4}$ Our data confirm those studies ${ }^{3,4}$ showing excellent results in terms of hospital mortality after RAVR, provided that endocarditis was not present, ${ }^{14}$ with EuroSCORE similarly affecting long-term rather than hospital mortality in our experience. Indeed, EuroSCORE proved to be an independent determinant of the composite endpoint of follow-up death and acute heart failure in our study.

When subgroups at higher risk were considered, it was noteworthy that elderly patients older than age $>75$ years demonstrated similar in-hospital and long-term outcomes 
NYHA IV
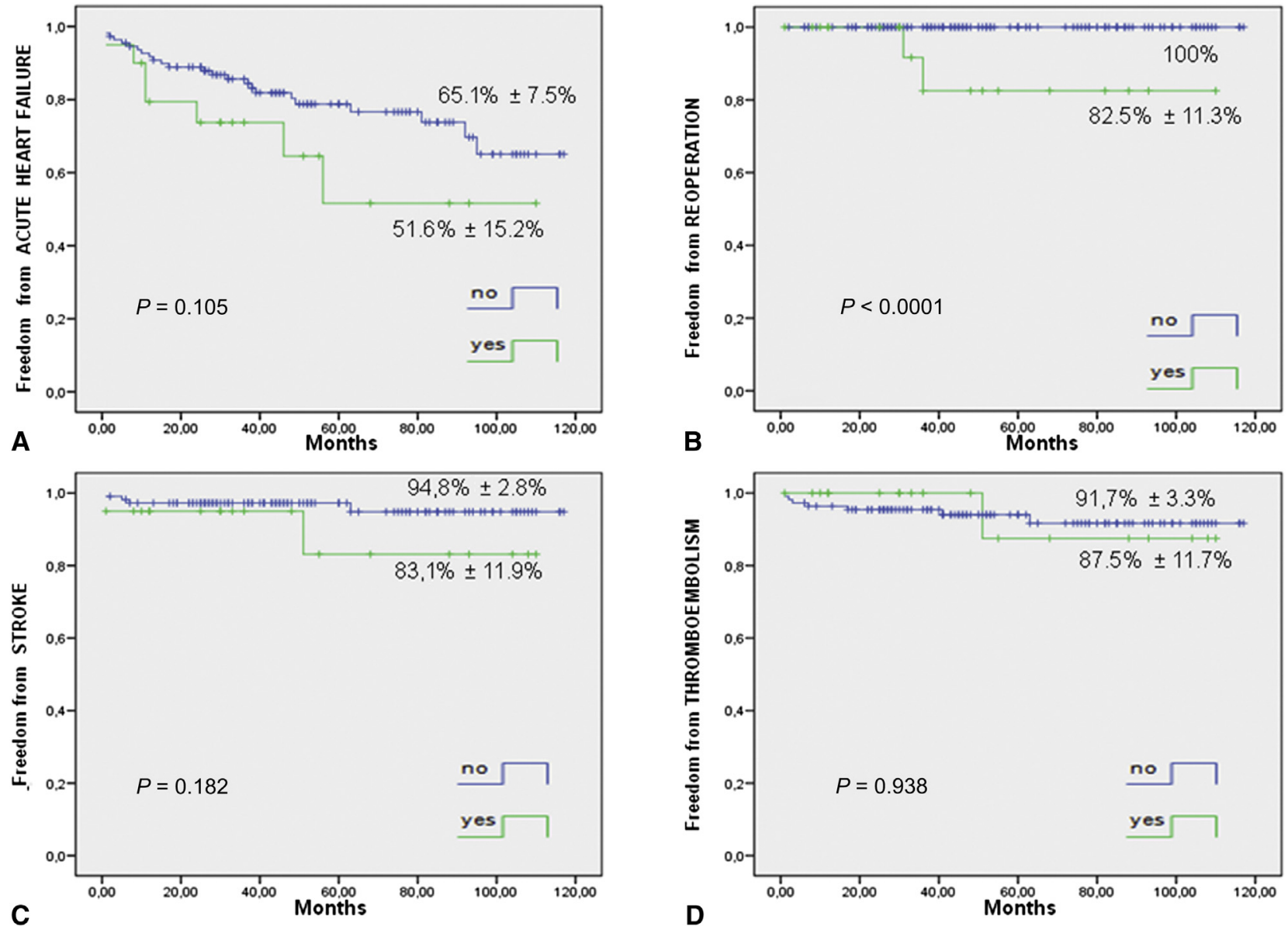

FIGURE 2. Ten-year actuarial freedom from (A) acute heart failure, (B) reoperations, (C) stroke, and (D) thromboembolisms in New York Heart Association class IV patients.

compared with younger patients. It is well known that cardiac surgery can be safely done in the last decades of life. ${ }^{20}$ Our data also supported the concept that EuroSCORE overestimates hospital mortality in aortic valve surgery, ${ }^{21}$ particularly in elderly patients. ${ }^{20}$ On the other hand, young patients aged $<65$ years showed a remarkably good long-term outcome in terms of survival and acute heart failure, superior in terms of absolute numbers-although not statistically significant- to that of elderly patients, thus supporting the preferential need for conventional RAVR in this subset of patients, especially with the current absence of long-term data after TAVIV.

When patients admitted in NYHA functional class IV and urgent/emergent indication were considered, a doubled hospital mortality, and a significantly higher incidence of low cardiac output syndrome, need for intra-aortic balloon pump, prolonged ventilation, and the related consequent higher incidence of pneumonia, were detected in NYHA functional class IV, suggesting the potential for a better outcome with TAVIV in this high-risk cohort, as early as during hospitalization. Accordingly, NYHA functional class at admission proved to be an independent predictor of the composite endpoint of death and/or acute heart failure at long-term follow-up. NYHA functional class is a well-recognized risk factor in both EuroSCORE and Society of Thoracic Surgeons score: A recent analysis identified a 10-fold higher hospital mortality, but similar long-term survival, in patients admitted in NYHA functional class III/IV. ${ }^{22}$ Our data confirm either worse hospital outcome or overall satisfactory long-term prognosis in NYHA functional class IV hospital survivors. $^{22}$ Moreover, our study demonstrated that NYHA functional class I and II at admission identifies a subgroup of patients with remarkably good early and long-term outcomes. Our data suggest that alternative procedures to surgery should not be indicated in that peculiar cohort of patients, given the excellent outcome with conventional surgery.

Emergent/urgent procedures showed a significantly higher need for postprocedural permanent pacemaker implantation only early after surgery. Unstable hemodynamics, hemodynamic compromise and active 
URGENCY / EMERGENCY
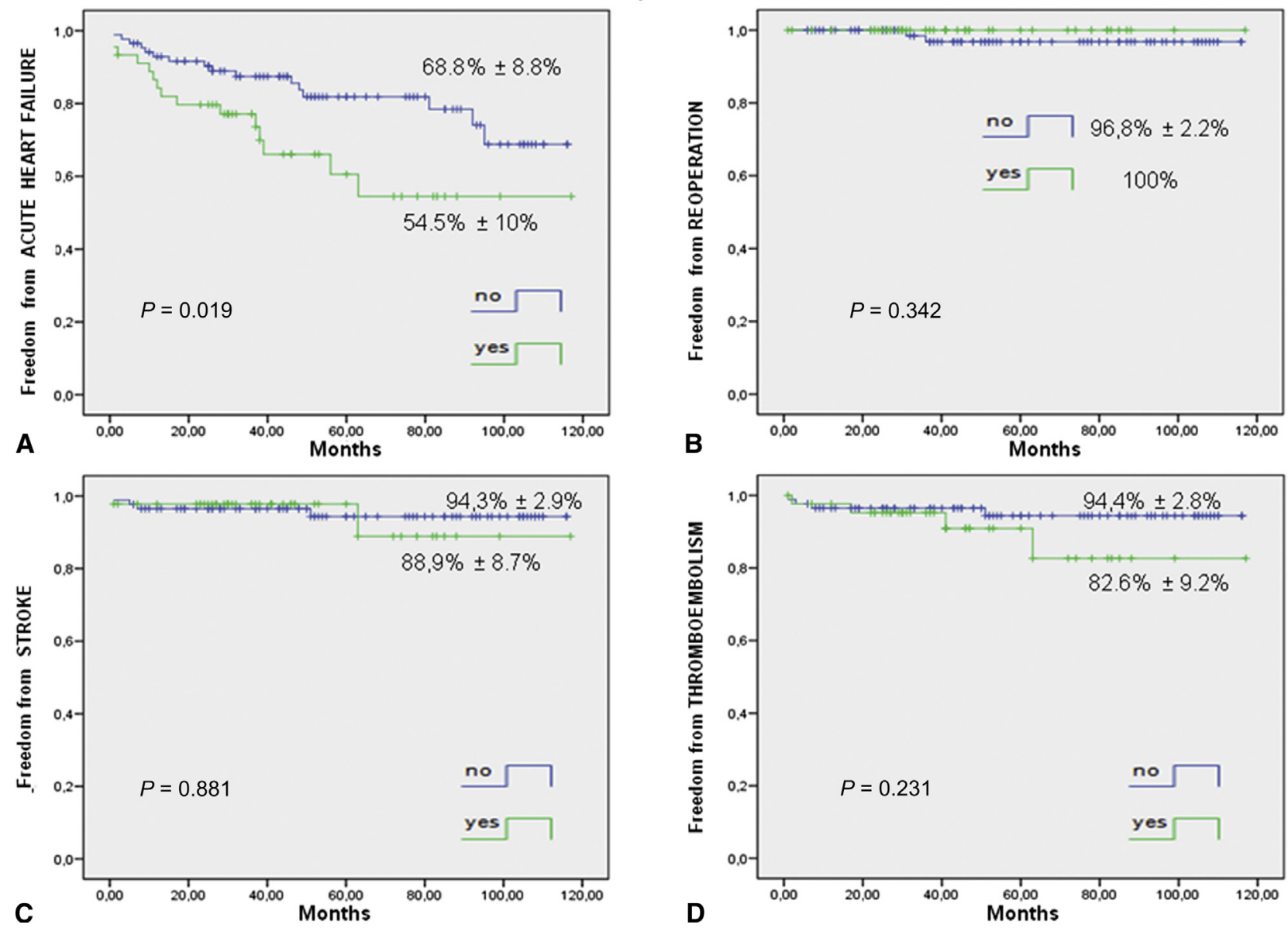

FIGURE 3. Ten-year actuarial freedom from (A) acute heart failure, (B) reoperations, (C) stroke, and (D) thromboembolisms in urgent/emergent procedures.

complicated endocarditis often are responsible for urgent/ emergent indications to surgery and are contemporarily responsible for the development of a complete atrioventricular block, mandating need for permanent pacemaker implantation. It has been demonstrated that pulmonary hypertension and intraoperative/postoperative cardiac arrest-all proxy variables of cardiac decompensation and unstable hemodynamics-predicted the need for permanent pacemaker after aortic valve replacement. ${ }^{23}$ Furthermore, cumbersome endocarditic procedures-extending deep in the annulus and involving the conduction system - may also help to explain these findings. $^{23,24}$ Furthermore, the profound affect of endocarditis on emergent/urgent procedures, observed also in our experience, help to explain the higher acute heart failure at follow-up in these patients, ${ }^{10,25}$ supporting the hypothesis of a potentially better outcome after TAVIV in patients with nonelective priority. Certainly future studies on TAVIV practice with longterm data in these peculiar subgroups of patients are needed before definitive conclusions.
Finally, our data confirm the already reported poor outcome for prosthetic endocarditis, ${ }^{10,25}$ which also proves as independent predictor of long-term mortality (the strongest predictor) and acute heart failure in our experience. Leontyev and colleagues ${ }^{10}$ have recently reported a significantly worse baseline profile, more technically demanding operations, worse hospital outcome (low output state, need for intra-aortic balloon pump, renal failure, and mortality), together with significantly lower 1-year $(52 \%)$ to 10 -year $(31 \%)$ survival, and an overall 5-year freedom from recurrences of $80 \%$ in endocarditis. ${ }^{10}$ Romano and colleagues $^{25}$ reported $24 \%$ hospital mortality and $33 \%$ 10 -year survival in prosthetic endocarditis. Similarly, endocarditis was the strongest predictor of the composite endpoint of follow-up death/acute heart failure in our population. Opposite to that, another interesting finding of our endocarditic-related subanalysis stem from the excellent results achieved by nonendocarditis patients, who demonstrated $<1 \%$ hospital mortality, rare periprocedural complications, $75 \%$ survival, $100 \%$ freedom from reoperation, and $93 \%$ freedom from stroke at 10 years, 
ENDOCARDITIS
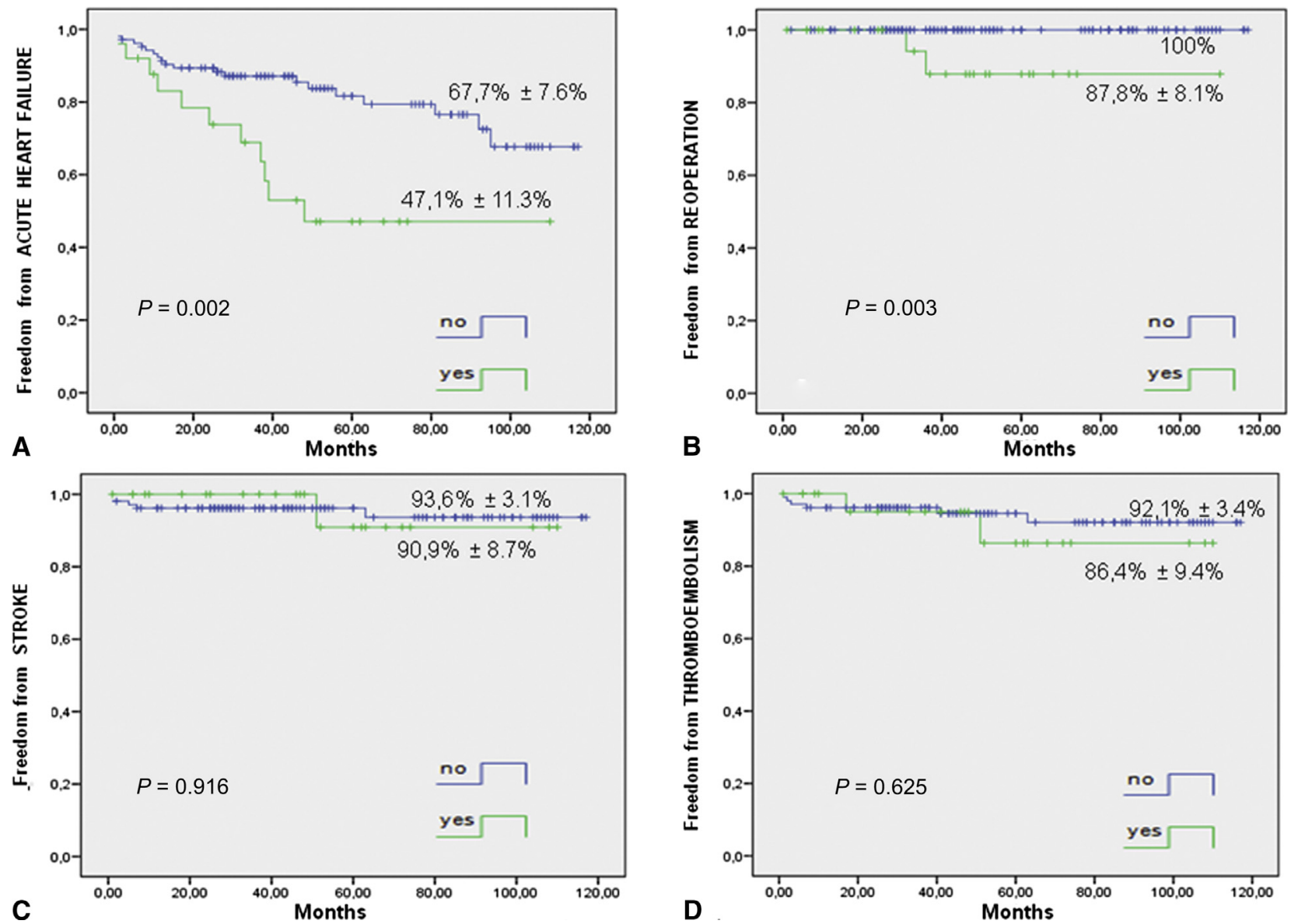

FIGURE 4. Ten-year actuarial freedom from (A) acute heart failure, (B) reoperations, (C) stroke, and (D) thromboembolisms in endocarditis.

and who represent nowadays the real redo population eligible for transcatheter aortic valve replacement. Therefore, despite endocarditis is obviously an absolute indication to surgery, also the absence of endocarditis can be useful for risk prediction, because it anticipates a good hospital and long-term follow-up outcome, especially if patients with low NYHA functional class or elective indication are considered. In our opinion, these patients should be first considered for surgery, rather than alternative procedures.

\section{Limitations of the Study}

The main limitation of our study is related to the single-center retrospective design. However, the literature lacks studies investigating current trends in RAVR in patients with long-term outcome data, without excessive case mix (eg, root procedures and concomitant mitral disease). Furthermore, the reported cohort of patients comprised also patients with endocarditis and with mechanical AVR at the time of the first operation who were not amenable to TAVR, so we decided to review our overall experience of RAVR to avoid selection bias and to report current results of all-comers undergoing RAVR at our institution during the past 11 years with at least 18-month follow-up. Another limitation is related to the absence of a direct comparison with a contemporary cohort of TAVIV patients; however, TAVIV is not the standard of care in our institution, and it is actually limited to a few patients judged to be contraindicated to RAVR by the local heart team. Furthermore, TAVIV is an extremely recent practice, thus no long-term data are available in the literature.

\section{CONCLUSIONS}

There is an urgent need for direct comparisons between RAVR and TAVIV in the literature, with both randomized controlled trials and observational registries. At the same time, a note of caution seems necessary for TAVIV procedures in the absence of long-term results.

\section{References}

1. Bach DS, Radeva JI, Birnbaum HG, Fournier AA, Tuttle EG. Prevalence, referral patterns, testing, and surgery in aortic valve disease: leaving women and elderly patients behind? J Heart Valve Dis. 2007;16:362-9. 
2. Rankin JS, Hammill BG, Ferguson TB, Glower DD, O'Brien SM, DeLong ER, et al. Determinants of operative mortality in valvular heart surgery. $J$ Thorac Cardiovasc Surg. 2006;131:547-57.

3. Leontyev S, Borger MA, Davierwala P, Walther T, Lehmann S, Kempfert J, et al. Redo aortic valve surgery: early and late outcomes. Ann Thorac Surg. 2011;91: 1120-6.

4. Launcelott S, Ouzounian M, Buth KJ, Legarè JF. Predicting in-hospital mortality after redo cardiac operations: development of a preoperative scorecard. Ann Thorac Surg. 2012;94:778-84.

5. Davierwala PM, Borger MA, David TE, Rao V, Maganti M, Yau TM Reoperation is not an independent predictor of mortality during aortic valve surgery. J Thorac Cardiovasc Surg. 2006;131:329-35.

6. Kirsch EW, Radu NC, Mekontso-Dessap A, Hillion ML, Loisance D. Aortic root replacement after previous surgical intervention on the aortic valve, aortic root, or ascending aorta. J Thorac Cardiovasc Surg. 2006;131:601-8.

7. David TE, Feindel CM, Ivanov J, Armstrong S. Aortic root replacement in patients with previous heart surgery. J Card Surg. 2004;19:325-8.

8. Dvir D, Webb J, Brecker S, Bleiziffer S, Hildick-Smith D, Colombo D, et al. Transcatheter aortic valve replacement for degenerative bioprosthetic surgical valves: results from the global valve-in-valve registry. Circulation. 2012;126: 2335-44.

9. Ferrari E. Transapical aortic "valve-in-valve" procedure for degenerated stented bioprosthesis. Eur J Cardiothorac Surg. 2012;41:485-90.

10. Leontyev S, Borger MA, Modi P, Lehmann S, Seeburger J, Walther T, et al. Redo aortic valve surgery: influence of prosthetic valve endocarditis on outcomes. J Thorac Cardiovasc Surg. 2011;142:99-105.

11. Onorati F, Santini F, Menon T, Amoncelli E, Campanella F, Pechlivanidis K, et al. Leukocyte filtration of blood cardioplegia attenuates myocardial damage and inflammation. Eur J Cardiothorac Surg. 2013;43:81-9.

12. Akins CW, Miller DC, Turina MI, Kouchoukos NT, Blackstone EH, Grunkemeier GL, et al. Guidelines for reporting mortality and morbidity after cardiac valve interventions. J Thorac Cardiovasc Surg. 2008;135:732-8.

13. Wilbring M, Alexiou K, Tugtekin SM, Sill B, Simonis G, Matschke K, et al. Transcatheter valve-in-valve therapies: patient selection, prosthesis assessment and selection, results, and future directions. Curr Cardiol Rep. 2013;15:341.

14. Albu C, Swaans M, Ten Berg J. With the back against the wall: TAVI in a patient with endocarditis. Catheter Cardiovasc Interv. Oct 122012 [Epub ahead of print].
15. Webb JG, Wood DA, Ye J, Gurvitch R, Masson JB, Rodes-Cabau, et al. Transcatheter valve-in-valve implantation for failed bioprosthetic heart valves. Circulation. 2010;121:1848-57.

16. Eggebrecht H, Schafer U, Treede H, Boekstegers P, Babin-Ebell J, Ferrari M, et al. Valve-in-valve transcatheter aortic valve implantation for degenerated bioprosthetic heart valves. J Am Coll Cardiol Interv. 2011;4: 1218-27.

17. Pasic M, Unbehaun A, Dreysse S, Buz S, Drews T, Kukucka M, et al. Transapical aortic valve implantation after previous aortic valve replacement: clinical proof of the valve-in-valve concept. J Thorac Cardiovasc Surg. 2011; 142:270-7

18. Seiffert M, Franzen O, Conradi L, Baldus S, Schirmer J, Meinertz T, et al. Series of transcatheter valve-in-valve implantations in high-risk patients with degenerated bioprostheses in aortic and mitral position. Catheter Cardiovasc Interv. 2010;76:608-15.

19. Piazza N, Bleiziffer S, Brockmann G, Hendrick R, Deutsche MA, Opitz A, et al. Transcatheter aortic valve implantation for failing surgical aortic bioprosthetic valve: from concept to clinical application and evaluation. JACC Cardiovasc Interv. 2011;4:733-42.

20. Molstad P, Veel T, Rynning S. Long-term survival after aortic valve replacement in octogenarians and high-risk subgroups. Eur J Cardiothorac Surg. 2012;42: 934-40.

21. Siregar S, Groenwold RHH, de Heer F, Bots ML, van der Graaf Y, van Herwerden LA. Performance of the original EuroSCORE. Eur J Cardiothorac Surg. 2012;41:746-54.

22. Maciejewski M, Piestrzeniewicz K, Bielecka-Dabrowa A, Piechowiak M, Jaszewski R. Redo surgery risk in patients with cardiac prosthetic valve dysfunction. Arch Med Sci. 2011;7:271-7.

23. Limongelli G, Ducceschi V, D’Andrea A, Renzulli A, Sarubbi B, De Feo M, et al. Risk factors for pacemaker implantation following aortic valve replacement: a single centre experience. Heart. 2003;89:901-4.

24. Elahi M, Usmaan K. The bioprosthesis type and size influence the postoperative incidence of permanent pacemaker implantation in patients undergoing aortic valve surgery. J Interv Card Electrophysiol. 2006;15:113-8.

25. Romano G, Carozza A, Della Corte A, De Santo LS, Amarelli C, Torella M, et al. Native versus prosthetic valve endocarditis: comparison of clinical features and long-term outcome in 353 patients. J Heart Valve Dis. 2004;13 200-8. 
TABLE E1. Preoperative and operative characteristics of patients

\begin{tabular}{lc}
\hline \multicolumn{1}{c}{ Characteristic } & Result \\
\hline Age, y & $65.6 \pm 12.9$ \\
European System for Cardiac Operative Risk Evaluation & $8.2 \pm 1.9$ \\
$\quad$ score (additive) & \\
Women & $32.8(43)$ \\
Chronic obstructive pulmonary disease & $9.2(12)$ \\
Left ventricular ejection fraction (Simpson method), \% & $51.8 \pm 10.5$ \\
Previous aortic valve prosthesis & \\
$\quad$ Mechanical & $19.1(25)$ \\
$\quad$ Biological & $80.9(106)$ \\
Type of intervention & \\
$\quad$ Redo aortic valve replacement & $75.5(99)$ \\
$\quad$ Redo aortic valve replacement + coronary artery & $24.5(32)$ \\
$\quad$ bypass graft & \\
Type of prosthesis & \\
$\quad$ Biologic & $65.6(86)$ \\
Mechanical & $29.0(38)$ \\
Homograft & $5.3(7)$ \\
Aortic crossclamp time, min & $104.0 \pm 50.4$ \\
Cardiopulmonary bypass time, min & $147.3 \pm 72.7$ \\
\hline
\end{tabular}

Values are presented as mean \pm standard deviation or $\%$ (n). 